\title{
The prolyl isomerase Pin1 is overexpressed in human esophageal cancer
}

\author{
HUAWEI JIN $^{1 *}$, JIE JIANG $^{2 *}$, LIFANG SUN $^{1 *}$, FANGFANG ZHENG ${ }^{1}$, \\ CHENGYAN WU ${ }^{1}$, LIN PENG ${ }^{1}$, YUFEN ZHAO ${ }^{1}$ and XUEJI WU ${ }^{1}$ \\ ${ }^{1}$ Department of Chemical Biology and The Key Laboratory for Chemical Biology of Fujian Province, \\ College of Chemistry and Chemical Engineering, Xiamen University, Xiamen 361005; \\ ${ }^{2}$ Xiamen Hospital of Traditional Chinese Medicine, Xiamen 361009, P.R. China
}

Received April 4, 2011; Accepted August 17, 2011

DOI: $10.3892 / \mathrm{ol} .2011 .392$

\begin{abstract}
Peptidyl-prolyl isomerase Pin1 specifically catalyzes the cis/trans-isomerization of proline in the target sequence of phosphorylated Ser/Thr-Pro in over 50 critical regulatory proteins. Pin1 is abnormally overexpressed in a range of human cancers, including lung, breast, colon and prostate cancers. However, few reports of Pin1 overexpression are currently available in clinical samples. Therefore, we examined the expression of Pin1 and p53 in non-pathological human tissues and esophageal cancer tissues. In esophageal cancer tissues, Pin1 and p53 immunoreactivity was detected in cancer cells in 67 and 58\% of cases, respectively. Moreover, Pin1 and p53 immunoreactivity was significantly correlated with lymph node-positive disease and more advanced cancer stage. The results demonstrated that high expression levels of Pin1 correlated with high levels of p53. Therefore, Pin1 is suggested to play key roles in the regulation of esophageal cancer.
\end{abstract}

\section{Introduction}

The human Pin1 (EC 5.2.1.8) was identified in 1996 by yeast two-hybrid screens as a protein that binds and inhibits the toxicity of never-in-mitosis A (NIMA), a fungal mitotic kinase (1). Human Pin1 contains 163 amino acid residues and consists of a substrate-recognition WW domain in the $\mathrm{N}$-terminal and a C-terminal catalytic PPIase domain (2).

Correspondence to: Dr Xueji Wu or Professor Yufen Zhao, Department of Chemical Biology and The Key Laboratory for Chemical Biology of Fujian Province, College of Chemistry and Chemical Engineering, Xiamen University, Xiamen 361005, P.R. China

E-mail:wuxj@xmu.edu.cn

E-mail:yfzhao@xmu.edu.cn

${ }^{*}$ Contributed equally

Key words: peptidyl-prolyl isomerase, esophageal cancer, reverse transcription-polymerase chain reaction, immunohistochemistry
Subsequent studies found that Pin 1 specifically binds and isomerizes pSer/Thr-Pro motifs in a large and defined subset of phosphoproteins, which are usually the substrates of proline-directed protein kinases. Proline-directed phosphorylation plays an essential role in normal, as well as in malignant, cell proliferation (3-5).

Functionally, the phosphorylation-dependent cis/trans-isomerization mediated by Pin1 has a profound impact on cell events. Such a conformational change regulates the activities of its substrates including catalysis, protein-protein interaction, subcellular localization, protein dephosphorylation and stability (5-7). For instance, Pin1 is abnormally overexpressed in a range of human cancers, including lung, breast, colon and prostate cancers, and is considered a biomarker of poor prognosis (8-12).

Extensive studies on Pin1 have identified over 50 proteins as biological substrates of Pin1. These proteins include cell cycle-regulated proteins such as Raf-1, Cdc25, cyclin D1, cyclin E, GTP-binding protein Rab4, anti-apoptotic protein Bcl-2, transcription factors c-Jun, $\beta$-catenin, NF- $\kappa$ B, p53, c-Myc, p73, c-fos, and Alzheimer's disease-related proteins APP and Tau (13-18).

Pin1 regulates the cancer suppressor protein p53 via its WW domain by promoting p53's stability in response to DNA damage induced by genotoxic drugs, UV light and ionizing radiation $(19,20)$. Pin1 is capable of interacting with a number of pSer/Thr-Pro motifs in p53, including pSer33, pSer46, pThr81 and pSer315 (21-23). Binding of Pin1 mediates a p53 conformational change, which in turn enhances interaction between 553 and the checkpoint kinase 2 (Chk2) and subsequent p53 phosphorylation at Ser20. Interaction between p53 and Chk2 protects p53 from Mdm2-mediated ubiquitination, nuclear export and degradation. Accumulation of p53 in turn enhances its transcriptional activity towards the cell cycle inhibitor $\mathrm{p} 21$. This elevation eventually leads to a cell cycle checkpoint arrest in response to DNA damage $(24,25)$.

Therefore, Pin 1 and p53 are considered to be involved in the regulation of cancer progression. In the present study, we conducted a quantitative investigation on 110 esophageal cancer specimens and matching normal esophageal tissue to examine the levels of Pin 1 expression in esophageal cancer and normal esophageal specimens by means of immunohistochemistry and reverse transcription polymerase 
chain reaction (RT-PCR) to ascertain the effect of Pin1 on esophageal cancer pathogenesis and development.

\section{Materials and methods}

Patients and tissues. Prior informed consent for the following studies was obtained from all patients. A total of 110 cancer specimens and matching normal esophageal tissues were obtained in consultation with the surgeon and the pathologist at the Xiamen Hospital of Traditional Chinese Medicine, Xiamen, China, between September 2004 and November 2008. The tissues were obtained immediately following surgery. The tissues for RNA isolation were snap-frozen and stored at $-80^{\circ} \mathrm{C}$, and those for immunohistochemistry were fixed with $10 \%$ formalin and embedded in paraffin wax. The clinicopathological characteristics of the subjects are shown in Table I.

Immunohistochemistry. Sections ( $4 \mu \mathrm{m})$ of formalin-fixed, paraffin-embedded tissue samples were cut with a microtome and dried overnight at $37^{\circ} \mathrm{C}$ on a silanized slide. The protocol for the avidin-biotin-peroxidase complex method was followed for each sample. Samples were deparaffinized in xylene at room temperature for $30 \mathrm{~min}$, rehydrated with graded ethanol and washed in phosphate-buffered saline (PBS). The samples were then laced in $10 \mathrm{mM}$ citrated buffer ( $\mathrm{pH} \mathrm{6.0)}$ and boiled in a microwave for $10 \mathrm{~min}$ for epitope retrieval. Endogenous peroxidase activity was quenched by incubating tissue sections in $3 \%$ $\mathrm{H}_{2} \mathrm{O}_{2}$ for 10 min. The primary antibodies mouse Pin1 (Abnova Corp., Taipei, Taiwan) and mouse p53 (Santa Cruz Biotechnology, Santa Cruz, CA, USA) were used overnight at $4^{\circ} \mathrm{C}$, at dilutions of 1:300 and 1:200, respectively. The slides were washed and biotinylated goat anti-mouse secondary antibody (Santa Cruz Biotechnology) was applied for $1 \mathrm{~h}$. Following rinsing in PBS, the slides were treated using the peroxidase-labeled Vectastain Elite $\mathrm{ABC}$ kit, and the peroxidase was subsequently developed with a diaminobenzidine kit and counterstained with hematoxylin. Goat serum was used for the negative controls instead of the primary antibody for Pin1 and p53.

Specimens of immunohistochemical staining for Pin1 and p53 were evaluated in a semi-quantitative manner, which considers the intensity of the staining and the percentage of cells stained at each intensity. In each case, the intensity (weak, moderate or strong) and pattern (incomplete or complete) of nuclear and cytoplasmic staining, and the percentage of neoplastic immunoreactive cells (cut-off of $10 \%$ ) were evaluated. Tumors were scored as: score 0 , no appreciable staining or staining in $<5 \%$ of neoplastic cells; score $1+$, tumors with faint/barely appreciable incomplete nuclear and cytoplasmic staining in $5-25 \%$ of neoplastic cells; score $2+$, tumors with weak to moderate complete nuclear and cytoplasmic staining or containing $25-50 \%$ of neoplastic cells with moderate incomplete basolateral nuclear and cytoplasmic immunostaining; score $3+$, strong immunoreactivity of the entire nucleus and cytoplasm in $>50 \%$ of neoplastic cells or containing $>50 \%$ neoplastic cells with strong basolateral incomplete nuclear and cytoplasmic immunoreactivity. Tumors were classified as 0 or $1+$, 'negative' and $2+$ or $3+$, 'positive'.

Primers. Primer sequences were designed for the $\mathrm{qRT}$ assay using Primer Premier Analysis Software, version 5.0. To avoid
Table I. Clinicopathological characteristics of 110 esophageal cancer patients.

\begin{tabular}{lc}
\hline Parameters & No. of patients (N) \\
\hline Patients & 110 \\
Lymph node & \\
Positive & 65 \\
Negative & 45 \\
Differentiation & \\
Low & 30 \\
Middle & 69 \\
High & 11 \\
Stage & \\
I & 31 \\
II & 40 \\
III+IV & 39 \\
TNM classification & \\
T1 & 8 \\
T2 & 67 \\
T3 & 11 \\
T4 & 24 \\
T1+T2 & 75 \\
T3+T4 & 35 \\
Gender & \\
Female & \\
Male & 18 \\
Age & 92 \\
$<49$ & \\
$50-59$ & 17 \\
$60-69$ & 18 \\
$>70$ & 46 \\
\hline
\end{tabular}

possible amplification of contaminating genomic DNA, primers were designed so that each PCR product covered at least one intron. The primer sequences utilized were: Pin1 forward, 5'-TGATCAACGGCTACATCCAG-3'; reverse, 5'-CAAACGAGGCGTCTTCAAAT-3'; and GAPDH forward, 5'-CATGACA ACTTTGGTATCGTG-3'; reverse, 5'-GTGTCGCTGTTGAAGTCAGA-3'. GAPDH was amplified as an internal reference housekeeping gene for assessing the status of the mRNA sample.

Real-time RT-PCR assay. Total cellular RNA from tissue specimens was isolated using a TRIzol reagent (Gibco BRL Life Technologies, Grand Island, NY, USA) according to the manufacturer's instructions. The total RNA ( $3 \mu \mathrm{g})$ was reversed transcribed using Moloney murine leukemia virus reverse transcriptase and random primers (Promega, Madison, WI, USA).

Real-time PCR was carried out with an Opticon 2 Real-Time Thermocycler (two colors) detection system (MJ Research, Canada). The PCR reaction solution ( $25 \mu \mathrm{l})$ contained cDNA from $250 \mathrm{ng}$ of total RNA, $1.5 \mathrm{mM} \mathrm{MgCl}{ }_{2}, 0.2 \mathrm{mM} \mathrm{dNTP}$, $0.6 \mu \mathrm{M}$ of each primer, 1 unit Taq polymerase (Takara, Otsu, Shiga, Japan), $0.625 \mu 1$ 1X SYBR-Green1 (Molecular Probes, 

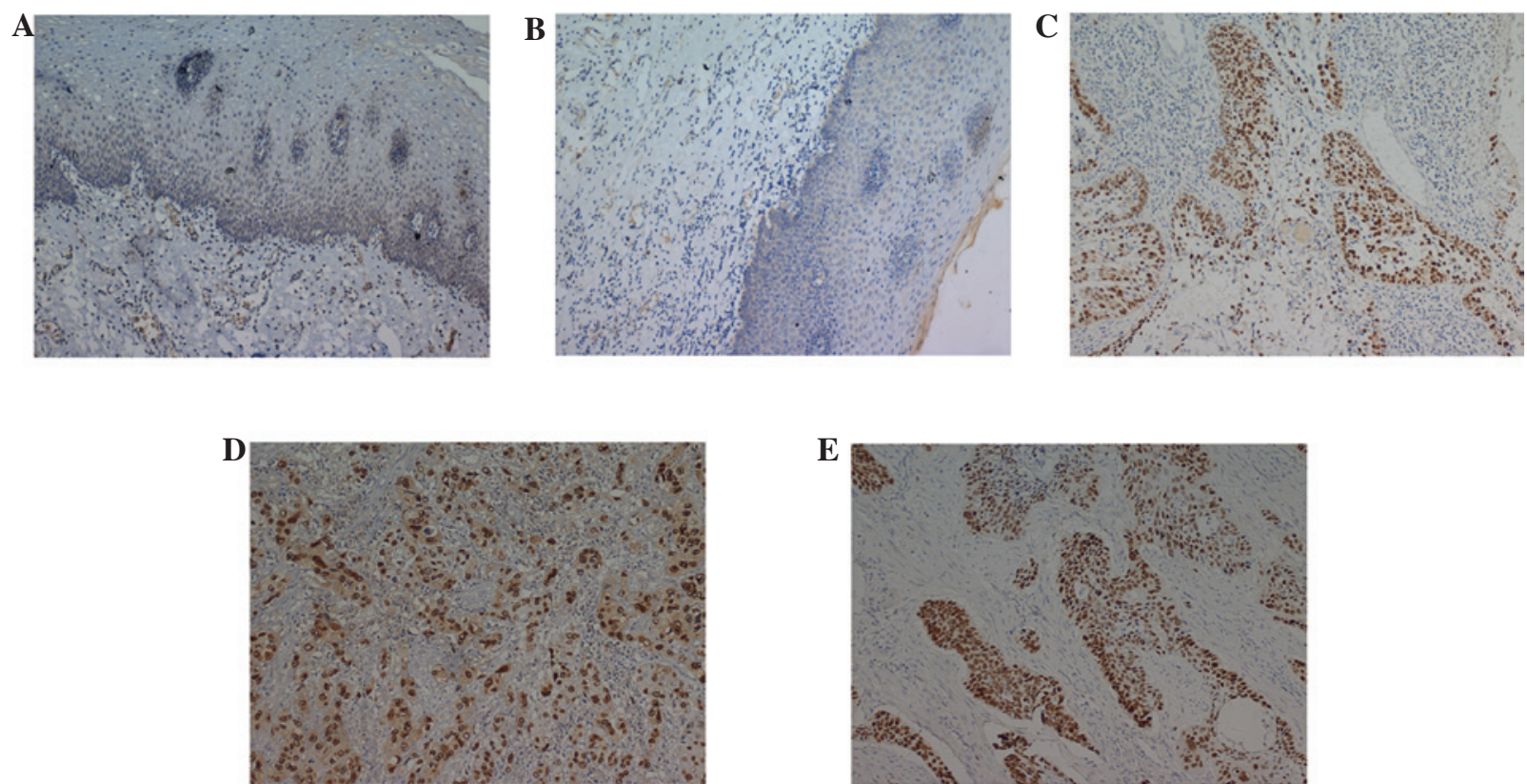

Figure 1. Representative immunohistochemical staining for Pin1 and p53. Sections from paraffin-embedded tissues were subjected to antigen retrieval treatment, followed by immunostaining with anti-Pin1 or anti-p53 antibodies. (A) Normal esophageal tissue with Pin1 immunostaining; (B) high differentiation of tumor tissue with Pin1 immunostaining; (C) middle differentiation of tumor tissue with p53 immunostaining; (D) low differentiation of tumor tissue with Pin1 immunostaining; and (E) tumor tissue with p53 immunostaining. Magnification, x100. The specimens were evaluated independently by two pathologists in a blinded manner without previous knowledge of the qRT results on Pin1 mRNA expression. The immunohistochemistry results for Pin1 were arbitrarily classified into four scores dependent on the intensity of immunoreactivity as described in Materials and methods.

Eugene, OR, USA; dilution 1:1000) and $2.5 \mu 1$ 10X AmpliTaq buffer for the final volume. PCR was performed under the following conditions: samples were initially denatured by heating at $94^{\circ} \mathrm{C}$ for $5 \mathrm{~min}$, followed by 40 cycles of denaturation at $94^{\circ} \mathrm{C}$ for $30 \mathrm{sec}$ and annealing at $58^{\circ} \mathrm{C}$ for $30 \mathrm{sec}$ for the two genes, and extension at $72^{\circ} \mathrm{C}$ for $1 \mathrm{~min}$. Each assay was performed at least three times to verify the results.

The standard curve for quantifying mRNA copy number was established by amplifying six aliquots of templates with known copy numbers $\left(2.1 \times 10^{3}-2.1 \times 10^{8}\right.$ copies $)$. cDNA was synthesized as follows: RT-PCR on the sample RNA was performed, electrophoresis was run on a $2 \%$ agarose gel, the Pin1 cDNA and GAPDH cDNA were ligased separately into pCR II-TOPO cloning vectors (Invitrogen, San Diego, CA, USA), the cDNA clones were transformed into Escherichia coli DH5- $\alpha$ cells and the cultures were expanded as previously described (9). Plasmids containing the target gene were purified and quantified for use in the qRT setup. To confirm that the inserted PCR product size was correct, plasmids were digested with specific restriction enzymes, and the cDNA clone PCR products were then run on gel electrophoresis. Finally, the two plasmids were sequenced by another biology company (Invitrogen) to confirm the sequencing results.

Statistical analysis. Statistical analysis was carried out using the Kappa analysis. Results were considered significant when $\mathrm{P}<0.05$.

\section{Results}

This study set out to examine the protein expression level of Pin1 and p53. A total of 110 esophageal cancer specimens
A

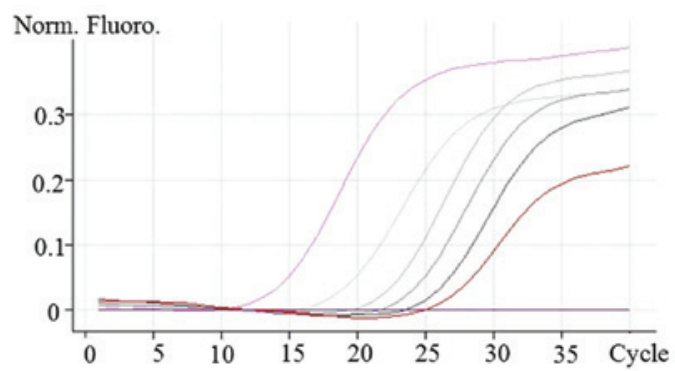

$\mathbf{B}$

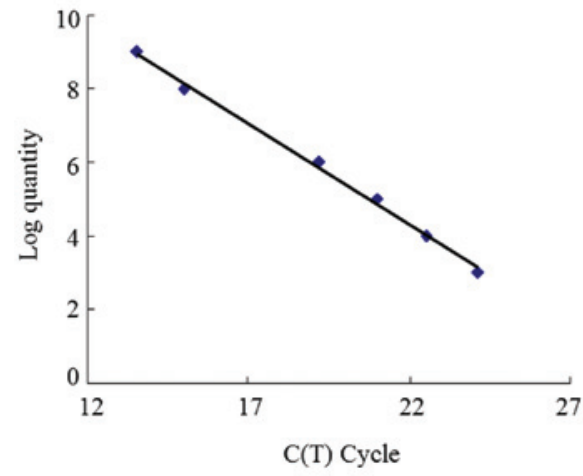

Figure 2. Representative q-RT analysis for Pin1 mRNA copy levels. (A) Serially diluted plasmids containing Pin1 cDNA $\left(2 \times 10^{3}-2 \times 10^{9}\right)$ were analyzed for controls. (B) Standard curve (correlation coefficient, 0.9969). Norm. Fluoro; normal fluorescence.

and matching normal esophageal tissues were analyzed by immunohistochemistry. Fig. 1 shows representative cancer tissue sections stained for the Pin 1 and $\mathrm{p} 53$ proteins. A number of clinicopathological categories were used to evaluate the 
Table II. The correlation between Pin 1 and p53 protein expression and clinicopathological characteristics.

\begin{tabular}{|c|c|c|c|c|c|c|c|}
\hline \multirow[b]{2}{*}{ Parameters } & \multirow[b]{2}{*}{$\mathrm{N}$} & \multicolumn{3}{|c|}{ Pin1 staining (IHC) } & \multicolumn{3}{|c|}{ p53 staining (IHC) } \\
\hline & & Positive & Negative & P-value & Positive & Negative & P-value \\
\hline & 110 & 74 & 36 & & 63 & & \\
\hline \multicolumn{8}{|l|}{ Lymph node } \\
\hline Positive & 65 & 54 & 11 & & 40 & 25 & \\
\hline Negative & 45 & 20 & 25 & 0.000 & 23 & 22 & 0.277 \\
\hline \multicolumn{8}{|c|}{ Differentiation } \\
\hline Low & 30 & 24 & 6 & & 22 & 8 & \\
\hline Middle & 69 & 45 & 24 & & 36 & 33 & \\
\hline High & 11 & 5 & 6 & 0.095 & 5 & 6 & 0.104 \\
\hline \multicolumn{8}{|l|}{ Stage } \\
\hline I & 31 & 18 & 13 & & 20 & 11 & \\
\hline II & 40 & 34 & 6 & & 22 & 18 & \\
\hline III+IV & 39 & 29 & 10 & 0.038 & 21 & 18 & 0.626 \\
\hline \multicolumn{8}{|c|}{ TNM classification } \\
\hline $\mathrm{T} 1$ & 8 & 7 & 1 & & 7 & 1 & \\
\hline $\mathrm{T} 2$ & 67 & 55 & 12 & & 35 & 32 & \\
\hline T3 & 11 & 8 & 3 & & 7 & 4 & \\
\hline $\mathrm{T} 4$ & 24 & 19 & 5 & 0.847 & 14 & 10 & 0.275 \\
\hline $\mathrm{T} 1+\mathrm{T} 2$ & 75 & 62 & 13 & & 42 & 33 & \\
\hline $\mathrm{T} 3+\mathrm{T} 4$ & 35 & 27 & 8 & 0.492 & 21 & 14 & 0.693 \\
\hline \multicolumn{8}{|l|}{ Gender } \\
\hline Female & 18 & 12 & 6 & & 12 & 6 & \\
\hline Male & 92 & 70 & 22 & 0.484 & 50 & 42 & 0.260 \\
\hline \multicolumn{8}{|l|}{ Age } \\
\hline$<49$ & 17 & 12 & 5 & & 11 & 6 & \\
\hline $50-59$ & 18 & 12 & 6 & & 11 & 7 & \\
\hline $60-69$ & 46 & 28 & 18 & & 26 & 20 & \\
\hline$>70$ & 29 & 18 & 11 & 0.868 & 15 & 14 & 0.833 \\
\hline
\end{tabular}

In each case, the intensity (weak, moderate or strong) and pattern (incomplete or complete) of nucleus and cytoplasm staining, and the percentage of neoplastic immunoreactive cells (cut-off of 10\%) were evaluated. Tumors were scored as follows: score 0 , no appreciable staining or staining in $<5 \%$ neoplastic cells; score 1+, tumors with faint/barely appreciable incomplete nucleus and cytoplasm staining in 5-25\% neoplastic cells; score $2+$, tumors with weak to moderate complete nucleus and cytoplasm staining or containing 25-50\% neoplastic cells with moderate incomplete nucleus and cytoplasm immunostaining; and score $3+$, strong immunoreactivity of the entire nucleus and cytoplasm $>50 \%$ neoplastic cells or containing $>50 \%$ neoplastic cells with strong nucleus and cytoplasm immunoreactivity. Tumors classified as 0 or $1+$ were considered 'negative' and those scored as $2+$ or $3+$ were classified as 'positive'.

expression of the targets and their potential correlation with the status of the disease. As shown in Table II, the protein level of Pin 1 was significantly higher in cancer tissue than in normal tissue. A favorable correlation was found between Pin1 expression and the stage of the disease. However, there significant correlation was observed between Pin 1 expression and histological type or the level of differentiation of the cancer. On the other hand, the results indicated that p53 was expressed more often in cancer tissue than in normal tissue.

To further examine the expression pattern of Pin 1 in esophageal cancer tissues, we investigated the mRNA expression of Pin1 by quantitative real-time-PCR (q-RT).
Total cellular RNA from tissue specimens was extracted for RT-PCR analysis. Among the total RNA extractions from 110 esophageal cancer specimens, only the RNA extractions from 40 specimens qualified for further RT-PCR analysis. We generated standard curves using known quantities of Pin1 prepared and confirmed by sequencing in our laboratory. A representative graph of the curves obtained from serial dilutions of Pin1 is shown in Fig. 2. The Pin1 mRNA was identified as 'overexpressed' if, when calibrated against GAPDH, the tumor had $\mathrm{a} \geq 1.8$ expression ratio compared to normal tissue. The level of mRNA was then compared to various clinicopathological characteristics. In accordance 
Table III. Correlation between Pin 1 mRNA expression and clinicopathological characteristics.

\begin{tabular}{|c|c|c|c|c|}
\hline \multirow[b]{2}{*}{ Parameters } & \multirow[b]{2}{*}{$\mathrm{N}$} & \multicolumn{3}{|c|}{ Pin1 mRNA (q-RT) } \\
\hline & & $\begin{array}{l}\text { Overexpression } \\
(\%)\end{array}$ & $\begin{array}{l}\text { Underexpression } \\
\qquad(\%)\end{array}$ & P-value \\
\hline & 40 & $16(40)$ & $24(60)$ & \\
\hline \multicolumn{5}{|l|}{ Diff } \\
\hline Low & 13 & 5 & 8 & \\
\hline Middle & 18 & 7 & 11 & \\
\hline High & 9 & 3 & 6 & 0.959 \\
\hline \multicolumn{5}{|l|}{ Stage } \\
\hline I & 9 & 2 & 7 & \\
\hline II & 12 & 7 & 5 & \\
\hline III+IV & 19 & 7 & 12 & 0.164 \\
\hline \multicolumn{5}{|c|}{$\begin{array}{l}\text { TNM } \\
\text { classification }\end{array}$} \\
\hline $\mathrm{T} 1$ & 3 & 0 & 3 & \\
\hline $\mathrm{T} 2$ & 26 & 8 & 18 & \\
\hline T3 & 5 & 4 & 1 & \\
\hline $\mathrm{T} 4$ & 6 & 2 & 4 & 0.099 \\
\hline $\mathrm{T} 1+\mathrm{T} 2$ & 29 & 8 & 21 & \\
\hline $\mathrm{T} 3+\mathrm{T} 4$ & 11 & 6 & 5 & 0.110 \\
\hline \multicolumn{5}{|l|}{ Gender } \\
\hline Female & 6 & 3 & 3 & \\
\hline Male & 34 & 11 & 23 & 0.403 \\
\hline \multicolumn{5}{|l|}{ Age } \\
\hline$<49$ & 6 & 2 & 4 & \\
\hline $50-59$ & 12 & 5 & 7 & \\
\hline $60-69$ & 15 & 6 & 9 & \\
\hline$>70$ & 7 & 3 & 4 & 0.985 \\
\hline
\end{tabular}

Diff, differentiation.

with the earlier comparison with the expression protein levels, high-level Pin1 mRNA was significantly correlated with the presence of lymph node metastasis and with the stage of disease. No significant correlation was found between Pin1 mRNA expression and the histological or differentiation types of the tumors, the size of the tumor, or between Pin1 expression and the age or gender of the patient. These results are shown in Table III. As shown in Table IV, no significant correlation between the level of Pin1 protein expression and Pin1 mRNA expression.

\section{Discussion}

Pin1 has been shown to play a significant role in numerous steps of oncogenic signaling pathways $(24,25)$. For example, Pin1 collaborates with Ras signaling to increase the transcriptional activity of c-Jun towards cyclin D1 $(24,25)$. Furthermore, Pin1 is involved in the DNA damage response through modulation
Table IV. Correlation between Pin 1 protein expression (IHC) and mRNA expression (q-RT).

\begin{tabular}{lcccc}
\hline & & \multicolumn{3}{c}{ Pin1 protein (IHC) } \\
\cline { 3 - 5 } & $\mathrm{N}$ & Positive & Negative & P-value \\
\hline Pin1 mRNA & & & & \\
$(\mathrm{q}-\mathrm{RT})$ & & & & \\
Positive & 24 & 19 & 5 & 0.757 \\
Negative & 16 & 12 & 4 & \\
\hline IHC, immunohistochemistry. & & & \\
\hline
\end{tabular}

of p53 function upon genotoxic stress $(24,25)$. Pin1 is overexpressed in human breast and oral cancers, and a high Pin1 expression is correlated with cancer development and poor prognosis in patients with prostate cancer $(24,25)$. However, whether or not there is any correlation between Pin 1 expression and the clinical outcome of cancer patients remains to be determined. To address this question, we determined Pin1 expression in 110 specimens using immunohistochemistry, followed by quantitative real-time-PCR.

This is the first large-scale study of Pin 1 expression in clinical samples of esophageal cancer. We have shown that Pin1 is overexpressed in esophageal cancer, and that its presence correlates with lymph node involvement and late stage disease. We also examined tumors for a correlation between Pin 1 expression and the expression of p53. In tumors with high levels of Pin1 expression, there was also a strong likelihood for the tumors to exhibit high levels of p53 expression. Since Pin1 is overexpressed in tumors, and since high levels of expression correlate with a poorer prognosis for patients, Pin1 may have the potential to be an excellent tumor marker.

Furthermore, an elevated Pin1 expression correlates with clinical stage. The Pin1 expression level may prove useful during biopsies, perhaps indicating a need for further treatment for patients with high Pin1 tumors. The strong correlation between the Pin1 level and clinical outcome of esophageal cancer suggests the involvement of Pin 1 in the progression of the disease. We have previously found that Pin1 is activated by oncogenic pathways via the transcriptional factor E2F, and that Pin1 overexpression activates multiple steps in oncogenic signaling pathways. No significant correlation was observed between the level of Pin1 protein expression and Pin1 mRNA expression. Therefore, this result would have suggested post-transcriptional regulation. Thus, our study establishes the role of Pin1 as a prognostic maker for biochemical recurrence in esophageal cancer and suggests that Pin1 is a novel target for treating patients with esophageal cancer.

\section{Acknowledgements}

This study was supported by the Scientific Research Foundation for Returned Overseas Chinese Scholars, the State Education Ministry (to X.W.) and the Xiamen Municipal Science and Technology Program (3502Z20064012 to J.J.). 


\section{References}

1. Lu KP, Hanes SD and Hunter T: A human peptidyl-prolyl isomerase essential for regulation of mitosis. Nature 380: 544-547, 1996.

2. Ranganathan R, Lu KP, Hunter T and Noel JP: Structural and functional analysis of the mitotic rotamase Pin1 suggests substrate recognition is phosphorylation dependent. Cell 89: 875-886, 1997.

3. Lu KP: Pinning down cell signaling, cancer and Alzheimer's disease. Trends Biochem Sci 29: 200-209, 2004.

4. Zhou XZ, Lu PJ, Wulf G and Lu KP: Phosphorylation-dependent prolyl isomerization: a novel signaling regulatory mechanism. Cell Mol Life Sci 56: 788-806, 1999.

5. Kim MR, Choi HS, Heo TH, Hwang SW and Kang KW: Induction of vascular endothelial growth factor by peptidyl-prolyl isomerase Pin1 in breast cancer cells. Biochem Biophys Res Commun 369: 547-553, 2008.

6. Ryo A, Liou YC, Wulf G, Nakamura M, Lee SW and Lu KP: PIN1 is an E2F target gene essential for Neu/Ras-induced transformation of mammary epithelial cells. Mol Cell Biol 22: 5281-5295, 2002.

7. Lu KP, Liou YC and Zhou XZ: Pinning down proline-directed phosphorylation signaling. Trends Cell Biol 12: 164-172, 2002.

8. Wulf GM, Ryo A, Wulf GG, et al: Pin1 is overexpressed in breast cancer and cooperates with Ras signaling in increasing the transcriptional activity of c-Jun towards cyclin D1. EMBO J 20: 3459-3472, 2001.

9. Bao L, Kimzey A, Sauter G, Sowadski JM, Lu KP and Wang DG: Prevalent overexpression of prolyl isomerase Pin1 in human cancers. Am J Pathol 164: 1727-1737, 2004.

10. Ayala G, Wang D, Wulf G, et al: The prolyl isomerase Pin 1 is a novel prognostic marker in human prostate cancer. Cancer Res 63: 6244-6251, 2003

11. He J, Zhou F, Shao K, et al: Overexpression of Pin1 in non-small cell lung cancer (NSCLC) and its correlation with lymph node metastases. Lung Cancer 56: 51-58, 2007.

12. Lu KP: Prolyl isomerase Pin1 as a molecular target for cancer diagnostics and therapeutics. Cancer Cell 4: 175-180, 2003.

13. Wulf G, Garg P, Liou YC, Iglehart D and Lu KP: Modeling breast cancer in vivo and ex vivo reveals an essential role of Pin1 in tumorigenesis. EMBO J 23: 3397-3407, 2004.
14. Lim J and Lu KP: Pinning down phosphorylated tau and tauopathies. Biochim Biophys Acta 1739: 311-322, 2005.

15. Brondani V, Schefer Q, Hamy F and Klimkait T: The peptidyl-prolyl isomerase Pin1 regulates phospho-Ser77 retinoic acid receptor alpha stability. Biochem Biophys Res Commun 328: $6-13,2005$

16. Liou YC, Ryo A, Huang HK, et al: Loss of Pin1 function in the mouse causes phenotypes resembling cyclin D1-null phenotypes. Proc Natl Acad Sci USA 99: 1335-1340, 2002.

17. Liou YC, Sun A, Ryo A, et al: Role of the prolyl isomerase Pin1 in protecting against age-dependent neurodegeneration. Nature 424: 556-561, 2003.

18. Balastik M, Lim J, Pastorino L and Lu KP: Pin1 in Alzheimer's disease: multiple substrates, one regulatory mechanism? Biochim Biophys Acta 1772: 422-429, 2007.

19. Bulavin DV, Saito S, Hollander MC, et al: Phosphorylation of human $\mathrm{p} 53$ by p38 kinase coordinates $\mathrm{N}$-terminal phosphorylation and apoptosis in response to UV radiation. EMBO J 18: 6845-6854, 1999.

20. Buschmann T, Potapova O, Bar-Shira A, et al: Jun NH2-terminal kinase phosphorylation of p53 on Thr-81 is important for p53 stabilization and transcriptional activities in response to stress. Mol Cell Biol 21: 2743-2754, 2001.

21. Radhakrishnan SK and Gartel AL: CDK9 phosphorylates p53 on serine residues 33, 315 and 392. Cell Cycle 5: 519-521, 2006.

22. D'Orazi G, Cecchinelli B, Bruno T, et al: Homeodomaininteracting protein kinase-2 phosphorylates p53 at Ser 46 and mediates apoptosis. Nat Cell Biol 4: 11-19, 2002.

23. Kurihara A, Nagoshi H, Yabuki M, Okuyama R, Obinata M and Ikawa S: Ser46 phosphorylation of p53 is not always sufficient to induce apoptosis: multiple mechanisms of regulation of p53-dependent apoptosis. Genes Cells 12: 853-861, 2007.

24. Wulf GM, Liou YC, Ryo A, Lee SW and Lu KP: Role of Pin1 in the regulation of $\mathrm{p} 53$ stability and $\mathrm{p} 21$ transactivation, and cell cycle checkpoints in response to DNA damage. J Biol Chem 277: 47976-47979, 2002.

25. Ryo A, Liou YC, Lu KP and Wulf G: Prolyl isomerase Pin1: a catalyst for oncogenesis and a potential therapeutic target in cancer. J Cell Sci 116: 773-783, 2003. 